\title{
Microdevices for Studies of Cultured Neural Networks
}

\author{
Jerome Pine \\ Caltech 256-48, 1200 E. California Blvd., Pasadena, CA 91125
}

\begin{abstract}
A cultured network has the advantages that the network is two-dimensional and easily observed, that the biochemical environment can be controlled, and that conventional electrodes as well as extracellular electrodes incorporated into the cultured substrate can be used to selectively stimulate and record from individual neurons in the network. It is possible to study small numbers of connected neurons, from a few to hundreds. This talk will describe two techniques, the multielectrode array and the silicon neurochip, and their application to long-term communication with a network by means of simultaneous recording or stimulation of many neurons.
\end{abstract}

\section{INTRODUCTION}

In 1978 I stopped doing research in High Energy Physics and spent a year intensively studying neurobiology. As a physicist, the idea of studying intact nervous systems, with hundreds of millions or even many billions of interconnected cells was very intimidating, so I was intrigued when I learned about cultured neural networks in a dish. In culture, networks can be grown which have from a few to thousands of neurons, in a two-dimensional geometry, easily visible with the phase contrast microscope, and easily accessible to physiological measurements and biochemical manipulations. I decided to learn how to study cultures electrophysiologically, and I had an idea. Instead of just one or two recording electrodes, there could be an array of many, embedded in the bottom of the culture dish, sensing nearby neurons by extracellular recording, and also able to selectively stimulate the culture.

Microfabrication using photolithography had become a common electrical engineering technique, so I set out to fabricate arrays of small electrodes on glass substrates that would become the bottom of culture dishes. And I learned to grow neurons in culture, with the help of expert neurobiologists. Figure 1 shows part of what I then called an "electric petri dish" - which now would be called a multielectrode array or MEA. The phase contrast photo shows part of a mass culture of sympathetic neurons, a few thousand in the dish, growing on my array of 16 electrodes. The neurons had been dissociated from the superior cervical ganglion of a newborn rat about eight days before the picture was made. They adhered to

CP501, Stochastic Dynamics and Pattern Formation in Biological and Complex Systems, edited by S. Kim, K. J. Lee, and W. Sung

(C) 2000 American Institute of Physics 1-56396-914-9/00/\$17.00 


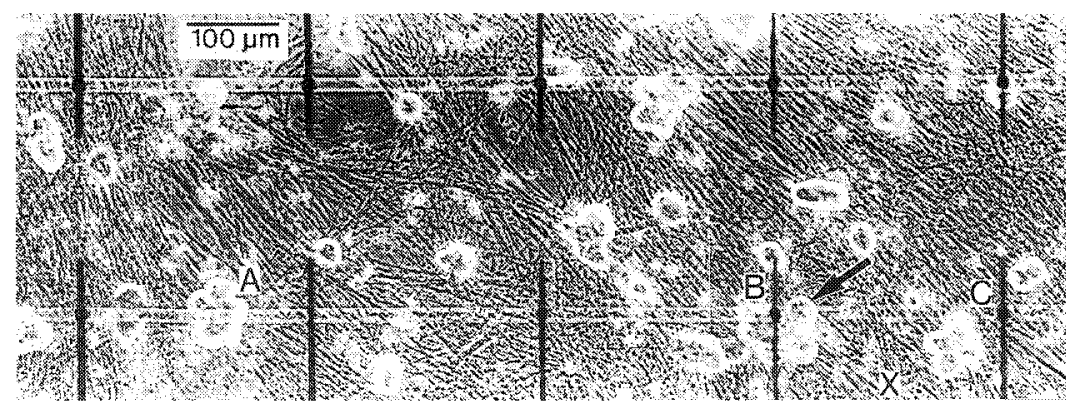

FIGURE 1. Cultured rat superior cervical ganglion neurons growing on a multielectrode array.

a polylysine treated surface and regrew their axons and dendrites to form a dense synaptically connected network, visible in the photograph.

The electrodes in the figure are black dots near the ends of gold leads which go up and down in the picture to terminals at the edge of the glass. The leads are covered with an insulating layer of silicon dioxide, and a horizontal groove which is visible in the figure near the ends of the leads was etched through to expose small 10 micron square gold areas. These were plated with platinum black to provide a low impedance electrical connection to the culture medium. Figure 2a shows a scanning electron micrograph of a platinized electrode, and the spongy deposit effectively increases the electrode surface area by one to two orders of magnitude.

Figure $2 \mathrm{~b}$ illustrates a recording made from the electrode near a cell labeled $\mathrm{B}$ with an arrow in Figure 1. The cell was penetrated with a sharp electrode, and short positive stimulus currents, of successively larger amplitude, were passed into

a

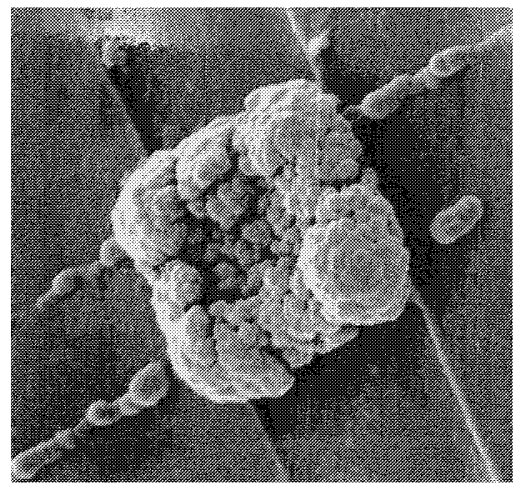

$\mathrm{b}$

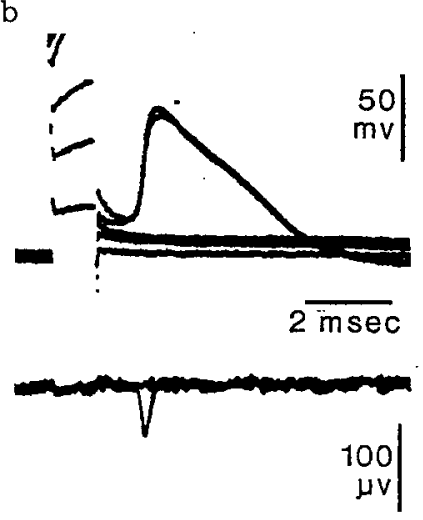

FIGURE 2. At left, a platinized electrode. At right, intracellular and extracellular recordings from a stimulated neuron. 

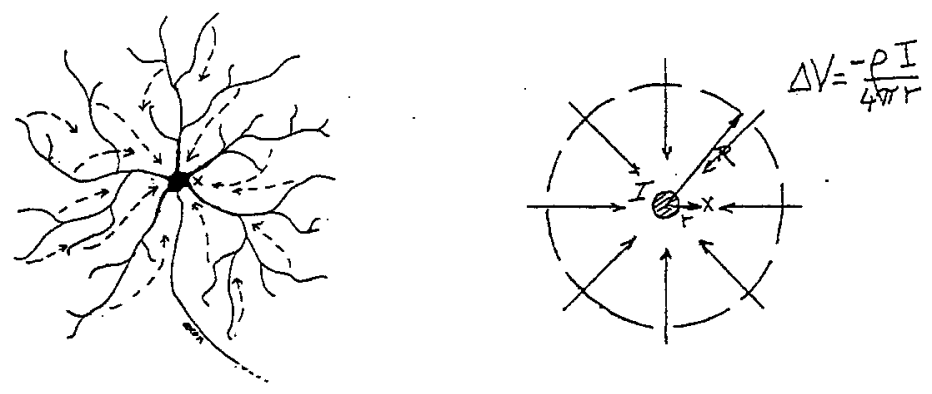

FIGURE 3. The biophysics of extracellular recording

the cell. The top traces show recordings from this electrode, which shows five successive stimuli and the cell response. When the stimulus exceeded a threshold, the cell fired an action potential about 70 millivolts high and 2 or 3 milliseconds wide. The extracellular electrode recording is shown on the bottom trace. The stimuli produce almost no response, but the action potentials are accompanied by sharp negative spikes. The signal to noise ratio is excellent, although the signals are only about 50 microvolts high. The noise from these electrodes and an inexpensive jfet preamp is negligible.

Figure 3 illustrates the biophysics of extracellular recording. At left is a schematic cell with an axon and many dendrites. When it fires an action potential a large current flows in through the sodium channels. The intracellular potential rises as shown in Figure 2, and current flows radially outward through the dendrites, leaking out through the membranes, and returning in current loops shown by the dashed arrows in the figure. If there is an extracellular electrode near the cell, as indicated by $\mathrm{x}$ in the figure, the potential at that point is lowered from that at the outer border of the dendrites by the ohmic voltage drop of the return current in the culture medium. The remote boundary of the dendritic tree is essentially at the "ground" reference potential of the medium, and a negative going signal with respect to ground will be seen at $x$. The signal is generated by current into the cell, and if the cell looks approximately like a "point sink" then the simple analysis diagrammed at the right in Figure 3 holds true, where I is the total current, $\rho$ is the resistivity of the culture medium, roughly $100 \mathrm{ohm}-\mathrm{cm}$, and $r$ is the distance from the center of the cell. Note that the large intracellular voltage changes generated by the stimulus currents are barely visible. It is the current into the cell that generates the signal, not capacitative current through the cell membrane. Inasmuch as this current into the cell is dominated by charging of the cell membrane capacitance, the current, and the extracellular signal, is approximately the derivative of the intracellular voltage.

During the summer of 1979 , when I was writing up experiments like the one just discussed [1], I searched the literature and learned that others before me had seen 
the possible benefit of an MEA in culture. In 1972, Thomas and his collaborators built an array for studying neurons but only succeeded in recording from heart cells [2]. In 1977, Gross and his colleagues [3] also built an array, but only used it for recording from the cells of an invertebrate ganglion. So the recordings I described were the first from dissociated neurons. Since then, Gross and many others have used arrays to record from neuron cultures.

\section{MEA APPLICATIONS}

In the past several years, a number of experimenters have studied mass cultures of mammalian brain neurons with MEAs. They have also laid brain slices over the arrays to record from them. Kanawa and his coworkers have done exceptionally interesting experiments with cultures [4,5] and brain slices [6], and he will speak about that work at this conference. I will therefore describe other MEA applications, with which we have been involved, and which I hope will illustrate a broad range of possibilities. The MEA described above evolved into a more well-developed device in our lab in the 1980s. Figure 4 illustrates the MEAs we built then, which were used for the experiments I will soon describe. The array is diagrammed in Figure $4 \mathrm{a}$, along with a schematic cross section, and consists of 61 electrodes spaced 70 microns apart. The spacing was chosen for experiments we wanted to do on small cultures of a few neurons, so that there would always be an electrode close enough to any neuron to record from it or stimulate it. The conductors are transparent indium tin oxide, on glass, and the exposed electrode areas are platinized by electroplating. The insulation was originally polyamide plastic, but has evolved to be silicon nitride or silicon dioxide, which are more durable. Figure $4 \mathrm{~b}$ shows the complete MEA assembly. The glass substrate is approximately $2 \times 4 \mathrm{~cm}$, and the the leads at the edge are connected to a printed circuit board with elastomer "zebra" connectors. The substrate is only $0.4 \mathrm{~mm}$ thick, to facilitate the use of
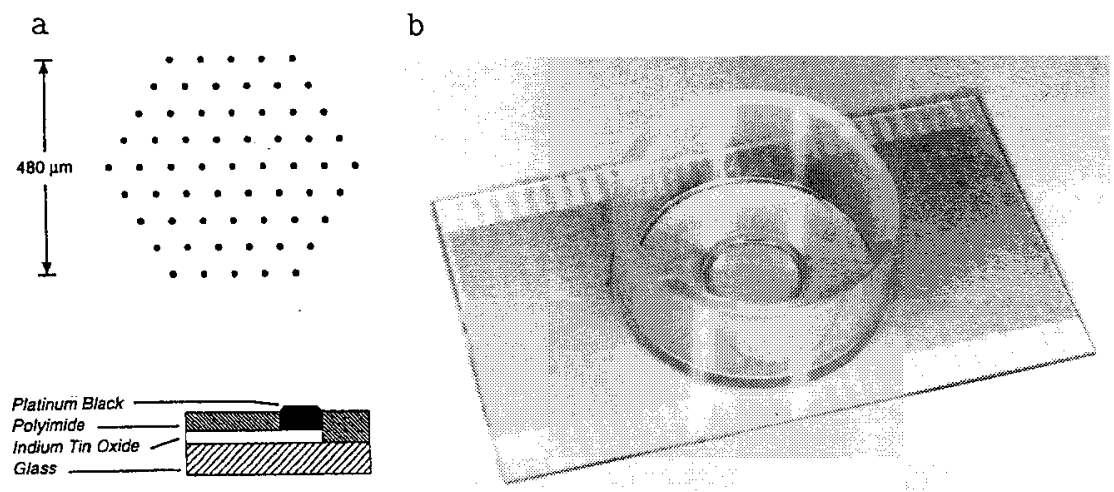

FIGURE 4. At left, the electrode structure, and at right the complete multielectrode array. 


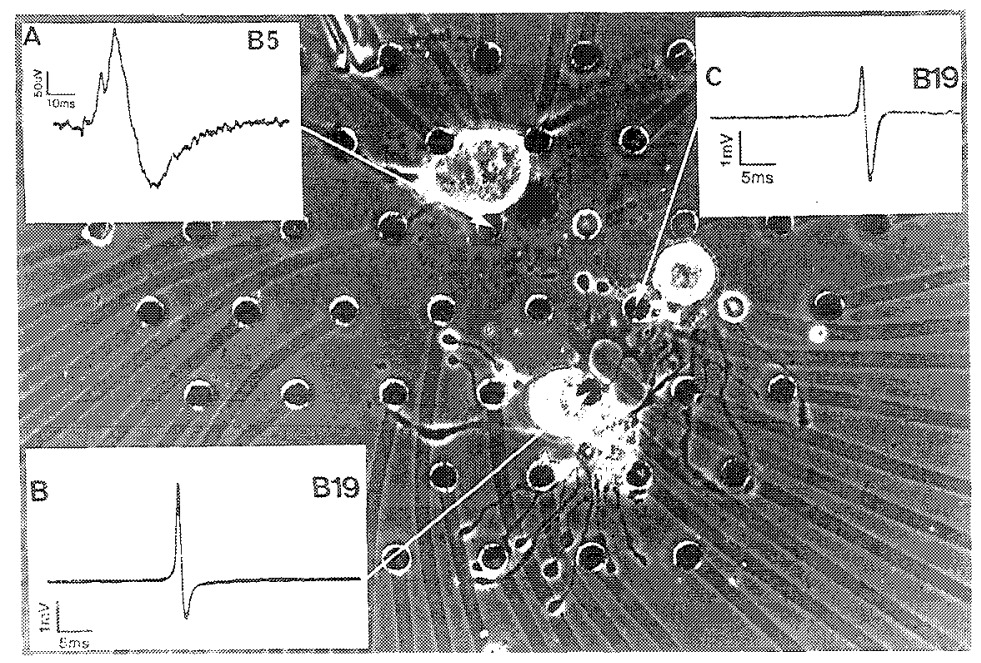

FIGURE 5. A network of spontaneously active snail neurons.

high magnification microscope objectives, which have short working distances.

One of the first studies done with our MEAs was the work of Wade Regehr and colleagues, to characterize the properties of MEAs for studies of large identified invertebrate neurons [7]. An example is shown in Figure 5, which shows recordings from a spontaneously active network of snail neurons. Note that the recordings from the two B19 neurons are quite different. In C, a conventional extracellular recording from a nearby neuron is seen, but in B there is a signal of opposite polarity. This signal illustrates the possibility of large capacitatively coupled signals from a neuron which has sealed over an electrode. For large invertebrate neurons, this is a potentially important effect, and Peter Fromhertz and collaborators have studied such signals, with large leech neurons placed over the gate of a field effect transistor [8]. The neurons in Figure 5 are typically 100 micrometers in diameter, but for the much smaller mammalian neurons this sealing technique is not promising.

Our interest has been focussed on the development and plasticity of small mammalian neural networks. To pursue our goal we want to determine the network connectivity by successively stimulating each neuron and recording the individual responses of all the others. We want to study the changes in connectivity over time during normal development, and then the effects on this connectivity of imposed patterns of stimuli. Such "plasticity" is believed to underly learning and memory, and our goal is to determine the basic rules. We also want to see small subtheshhold responses as well as action potentials, and these do not typically produce measurable extracellular signals, so we have therefore used voltage sensitive dyes to record them.

Figure 6a shows an extremely simple two-cell network and Figure $6 \mathrm{~b}$ shows the 
a

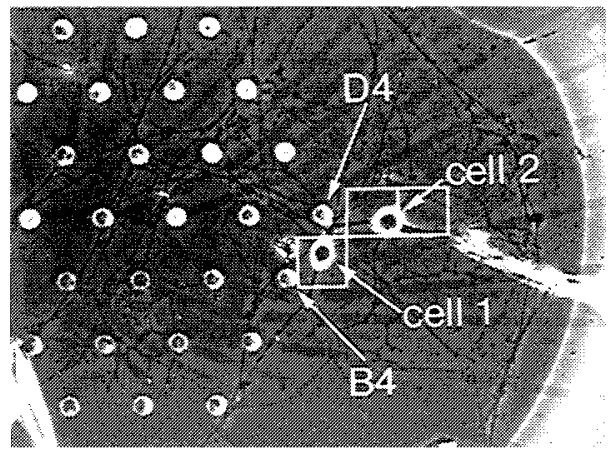

$\mathrm{b}$

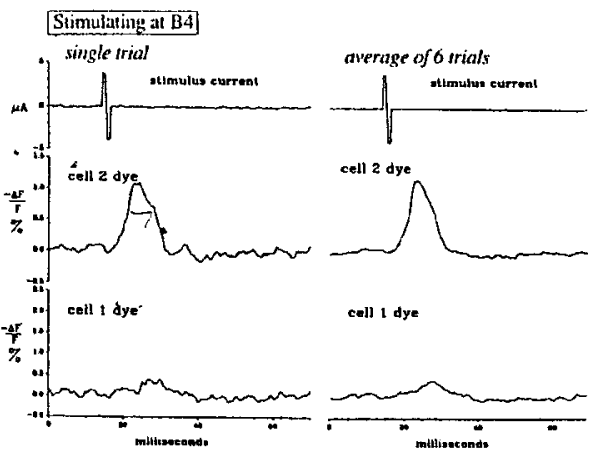

FIGURE 6. Stimulation of cell 2 and dye recordings, showing an action potential in 2 and a subthreshold synaptic potential in 1 .

dye recordings from a stimulation experiment. The stimulus current needed is roughly -10 microamperes, such that the ohmic drop near a stimulating electrode can produce a depolarization of $15 \mathrm{mv}$. or more on a nearby cell body or axon. The figure illustrates a serious difficulty, since in order to stimulate cell 2 and not cell 1 we had to search for an electrode, B4, rather remote from it. Otherwise, axons from both cells driven. For a more complex culture, selectively stimulating single cells will be very difficult. This led to our work with silicon structures in order to provide one-to-one communication with single neurons, which will be described later.

a

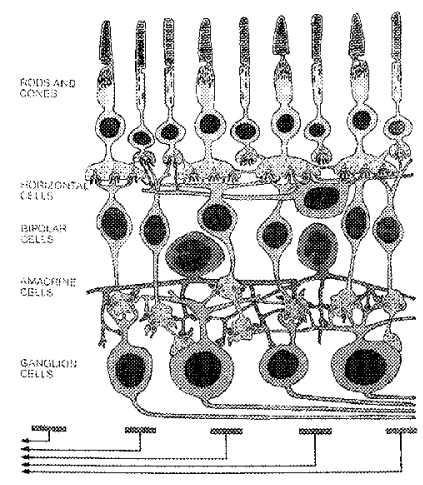

$\mathrm{b}$

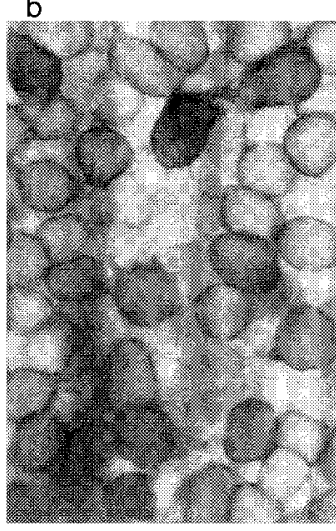

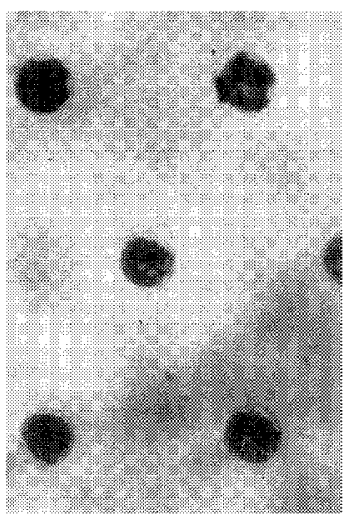

FIGURE 7. At left a schematic view of a retina, and at right the ganglion cells and array electrodes. 
a

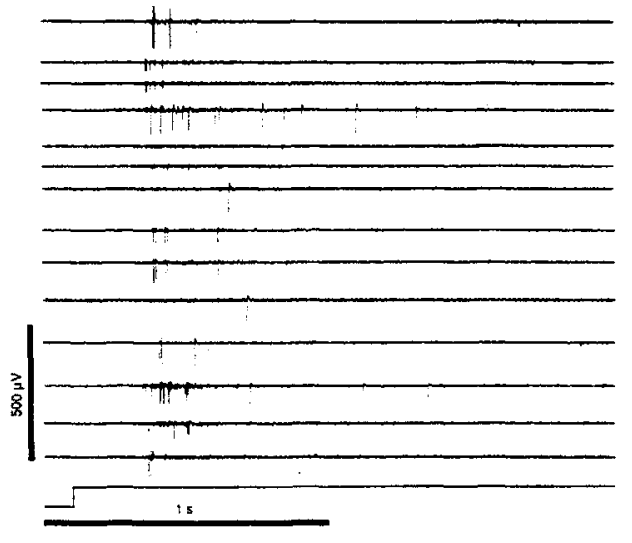

$\mathrm{b}$

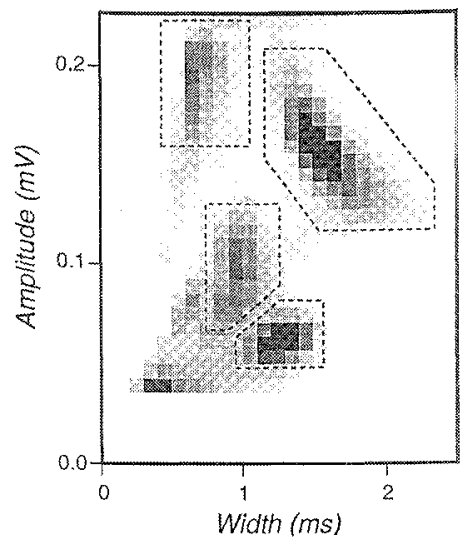

FIGURE 8. Recordings from a salamander retina, and separation of neurons on a single electrode.

Markus Meister suggested a very fruitful application of the MEA to a neural network dissected from a natural system: the retina. Figure 7a shows a schematic drawing of a mammalian retina, where photoreceptor signals are propagated, with complex signal processing, to output retinal ganglion cells which communicate with the brain. The ganglion cell layer of a dissected retina can be placed over an MEA, and the retina stimulated by light. Multineural signals recorded by the MEA can help reveal retinal signal processing in a way that is impossible with conventional single-cell electrophysology. Figure $7 \mathrm{~b}$ shows a view of stained salamander retinal ganglion cells over our array, and of the array alone. There are several cells per electrode, in a plane which is only tens of microns above the array. Figure 8a shows signals recorded simultaneously from 16 electrodes when the retina was exposed to a light stimulus, shown below the traces. As expected, many electrodes see signals from more than a single neuron, but it is usually possible to sort them simply by

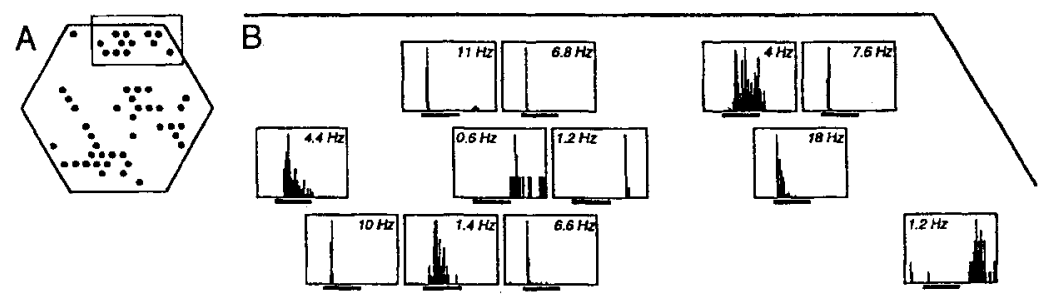

FIGURE 9. Firing patterns of multiple neurons stimulated by a light flash. 


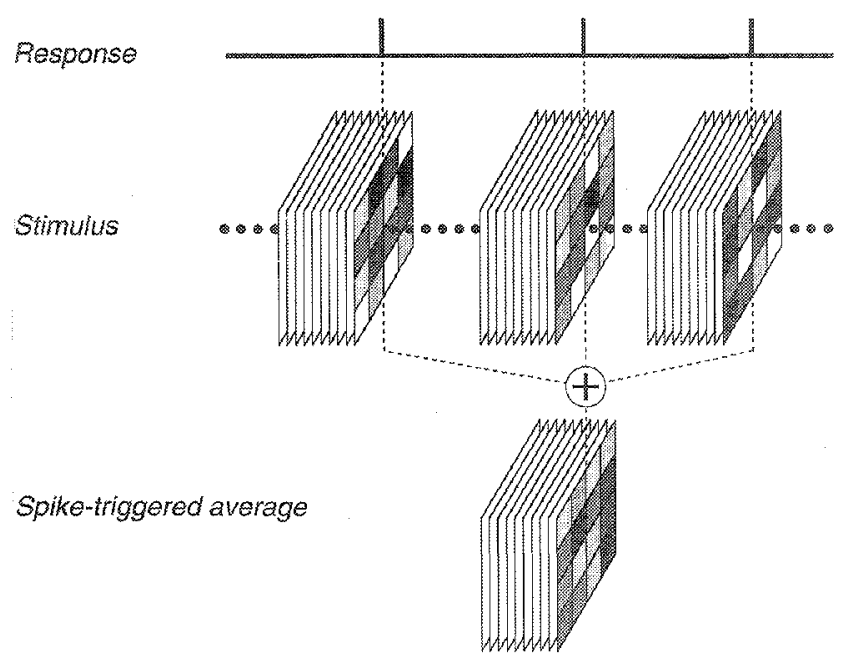

FIGURE 10. Spike-triggered averaging.

using the height and width of the action potentials. Figure $8 \mathrm{~b}$ shows a scatter plot with which four neurons recorded on a single electrode have been separated [9].

Figure 9 shows a sample from over 50 neurons recorded in one experiment, Firing rate vs. time histograms are shown, for data combined from many repeated two second long light stimuli, shown below the histograms. The numbers on the plot indicate maximum firing rates. It is clear that there are a great variety of responses to even this very simple stimulus, including neurons that respond to "on", to "off", or more steadily. Some show an amazing precision of timing, firing at a sharply defined time after the stimulus is turned on.

To pursue these studies further, Meister and collaborators have undertaken to measure the receptive fields of the ganglion cells, the retinal regions to which they respond. They used a very powerful technique based on illuminating a retina with a checker-board pattern of both intensity and color, randomly changing every 15 milliseconds. The method, spike-triggered averaging, is illustrated in Figure 10. For each spike, the stimuli during the preceding second are stored, and these are then averaged.

Figure 11 reveals the receptive field of a single neuron and its response time obtained in this way. The spike-triggered average of the random pattern, for a great many spikes, is calculated as a function of time before the spike. The display across the top of the figure shows the results, with a clear indication of the stimulus and its spatial extent at an optimal time 150 milliseconds before the spike. The larger view of this stimulus pattern has two rectangles marked, with the inside of the inner one interpreted as the excitatory receptive field, and the outside of the outer one as the surround. Sums over these regions as a function of time, 


\section{Mean Effective Stimulus for an ON cell}
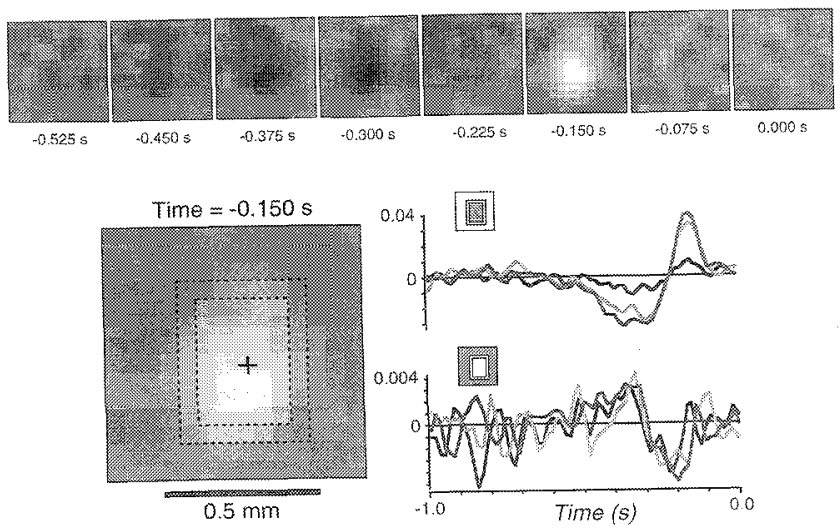

FIGURE 11. Receptive field, response time, and color response of a single ganglion cell.

for the three different color guns of the stimulus CRT, are shown at the lower right. The inhibitory effect of the surround is seen, as well as the spectral response and interesting "precurser" responses. With this powerful method, the receptive field, response time, and spectral sensitivity are simultaneously obtained for all the neurons recorded from. Meister and his coworkers are now engaged in ongoing studies of mammalian retinal processing.

An MEA application which led to an interesting result is the work of Welch and collaborators [10]. They grew suprachiasmatic neurons in a dissociated culture on our arrays. In vivo, these cells occupy a small nucleus in the brain and generate the 24-hour circadian clock which modulates nervous system activity. In the cultured neuron experiments, the dissociated cells spontaneously fired with approximately a 24 hour rhythm. Figure 12a shows the data from four electrodesi n one culture, recorded over a three day period. Figure $12 \mathrm{~b}$ shows the location of the electrodes from which the recordings were made. They were able to do long-term experiments spanning weeks, and the results showed that the neurons were not in phase, and longer term data indicated that their periods were not identical. The conclusion is that each individual suprachiasmatic neuron has its own independent 24 hour clock. The neurons were shown to be synaptically connected, but this did not synchronize them. At present, ongoing studies are focussed on the genetics and biochemistry of the clock.

\section{SILICON STRUCTURES}

About ten years ago, three-dimensional micromachining had become a maturing technology. We began to explore ways to use silicon microstructures for two-way 

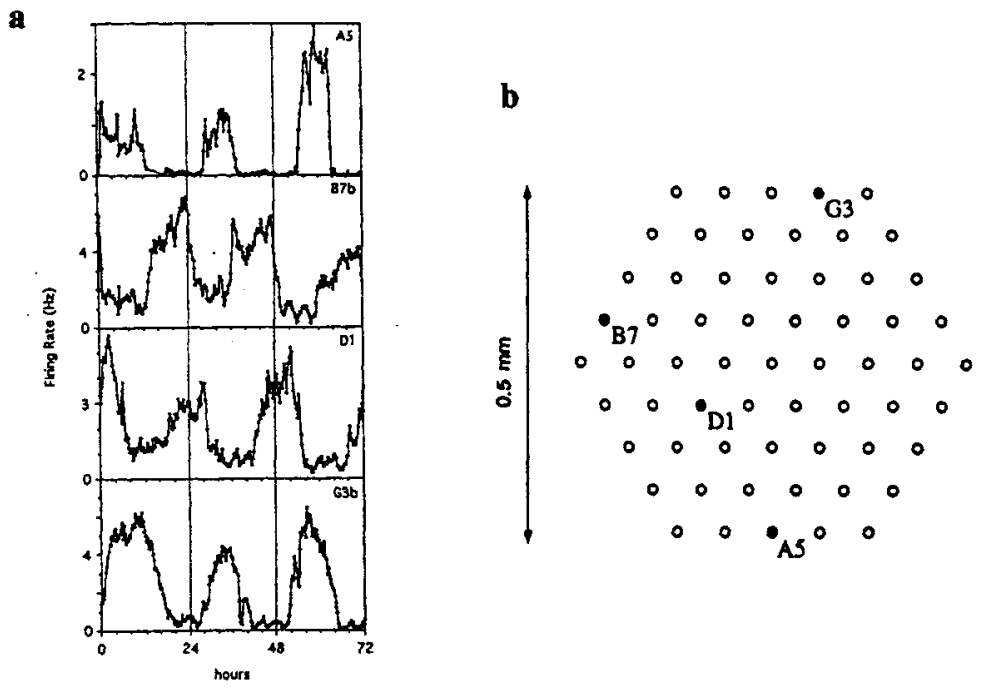

FIGURE 12. Spike rates for four suprachiasmatic neurons over a three day period, and the locations of the recording electrodes on the array.

communication with single neurons. The first experiments were designed to study the connectivity between an electrode in a cup-shaped structure and a neuron with which it was in contact. The idea was to see whether a seal might occur of the sort which occurs when patch-recording with glass pipettes. Figure 13 schematically illustrates a patch-pipette and a "diving board" microfabricated structure.

Wade Regehr made such diving board electrodes, with an arm 200 micrometers long, and one micrometer thick [11]. Fig. 14 is a scanning electron micrograph of the cup region of one electrode. Also shown is a diving board assembly suspended by its connecting lead. It could be "flown" down to a landing on a culture dish with the cup atop a neuron. UV-curing epoxy under the base of the board was

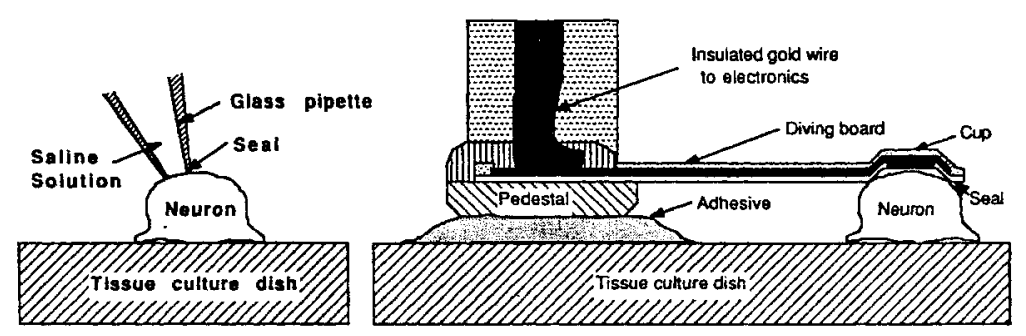

FIGURE 13. The concept of the diving board electrode. 

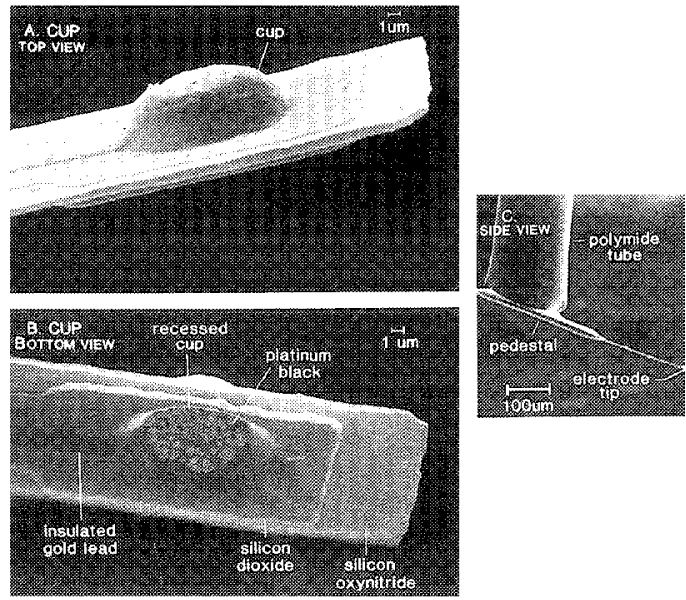

FIGURE 14. The cup of a diving board electrode; and the assembly.

used to attach it to the dish.

The result of this work were that good stimulation and recording could be achieved both on small mammalian neurons and on large invertebrate neurons. However, a tight seal was never achieved, and the electrical data indicated a gap of order $100 \mathrm{~nm}$ at the edge of the cup, which we ascribed to molecules on the outer surface of the neurons. The suction applied to a patch electrode to form a seal was not available for the diving board. Figure 15 shows a diving board being used to record over many days from a cultured snail neuron, and some of the cleanly recorded spontaneous activity is shown.

Whether or not we could obtain a patch recording, we embarked on a program to build a form of multielectrode array on a silicon chip, in which the electrodes were at the bottom of cavities which could each contain a single neuron. This would give

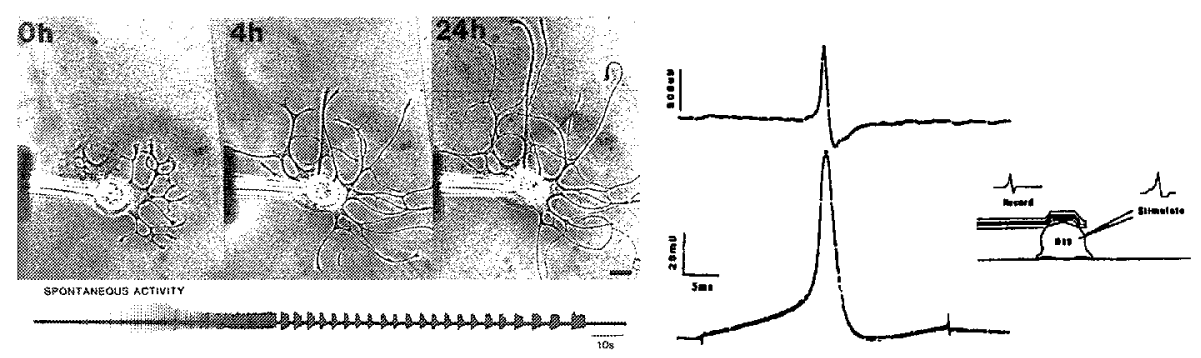

FIGURE 15. A diving board electrode on a snail neuron., with a recording from intracellular stimulation and a recording of spontaneous activity. 
a

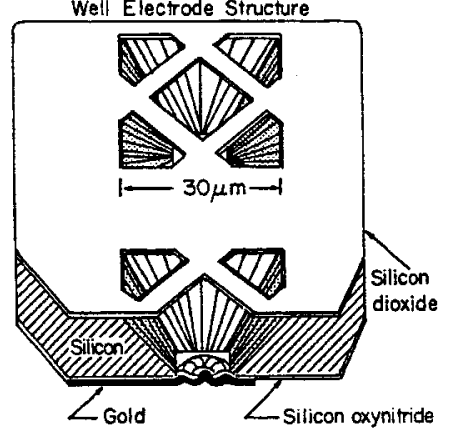

b

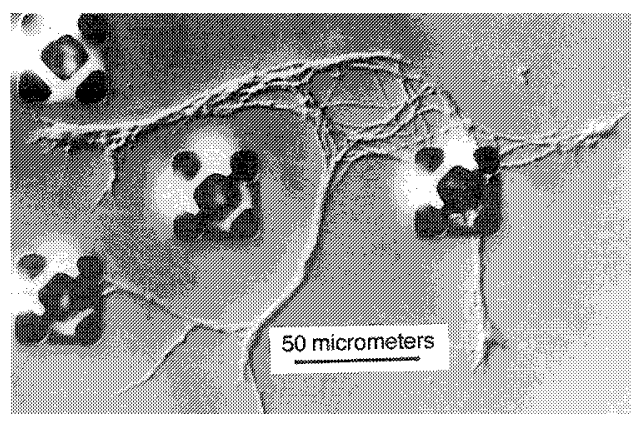

FIGURE 16. Neurowell concept, and successful outgrowth test.

the specificity of two-way communication needed for the network studies we wanted to do. Figure 16a shows the concept of the "neurowell" which we developed. Using anisotropic etching, the trapezoidal well could be made, and an etched insulating grillwork could cover it. The purpose of the grillwork was to prevent escape of a neuron after it had been loaded into a well. We envisioned the axons and dendrites growing out through the corner holes, thereby trapping the neuron. To begin, such wells were made on a thick silicon wafer, with no electrodes, to determine whether neurons would survive in them and grow out as we hoped. Figure 16b shows a sympathetic neuron about twelve hours after being placed in a well. The outgrowth is normal, and the neuron is indeed trapped.

Knowing that neurons would grow out of wells, the next step was to create a "neurochip" with many wells and electrodes in the bottom of the wells. We collaborated with Prof. Yu-Chong Tai of the Caltech Electrical engineering department who,

a

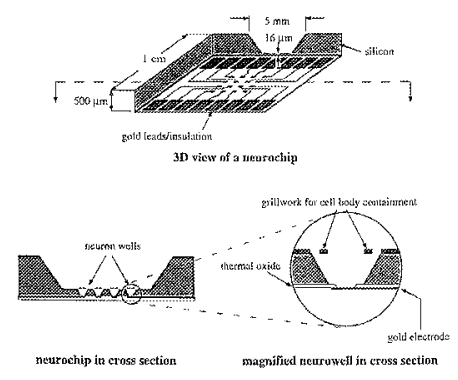

b

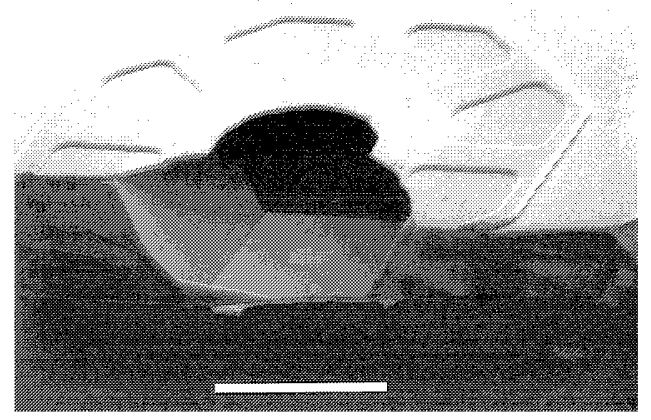

FIGURE 17. Schematic view of a neurochip. Scanning electron micrograph of a well. 


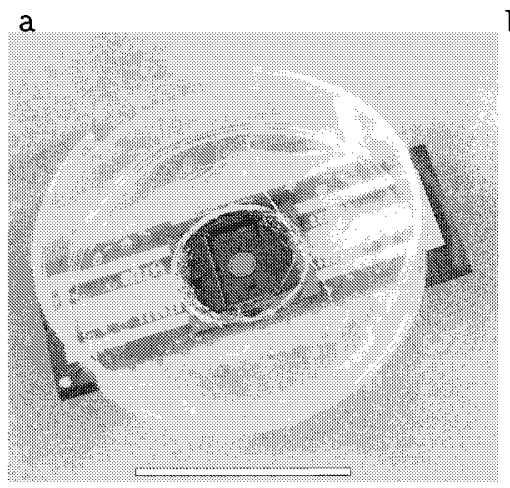

b

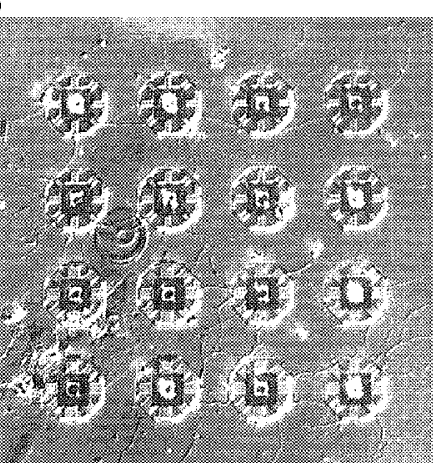

FIGURE 18. A neurochip assembly. A twelve cell network growing on a neurochip.

with his student John Wright, designed and executed the microfabrication. The major challenge was that the neurochip needed to have grillwork on one side and electrodes on the other side of a silicon membrane about 15 micrometers thick. Figure 17a shows the neurochip schematically. We planned for the initial experiments to have 16 wells in a $4 \times 4$ array. Anisotropic etching and projection lithography were used to pattern a membrane about $4 \times 8 \mathrm{~mm}$ in area. However, when we put hippocampal neurons in wells like the ones in Figure 16 we were chagrined to discover that they escaped within days. They grew axons and dendrites out the corners, and the cell then "climbed up " the axon and escaped. In retrospect, this was seen to emulate the behavior of these cells during development, when they migrate to their final locations. The sympathetic neurons we had been using for initial tests do not behave like that.

We did a series of experiments with smaller and smaller grillwork holes to try to control escape, but these were not successful. Finally, Michael Maher from our lab and John Wright, from the Tai lab, invented the idea of wells with tunnels. Figure $17 \mathrm{~b}$ is a scanning micrograph of such a well, which has been broken to reveal its cross section. The scale bar is 20 micrometers long. The anisotropically etched well, with an electrode visible at the bottom, is under an octagonal silicon nitrode canopy, 0.5 micrometers thick, with a central hole through which a cell can be loaded. The canopy is raised 0.5 micrometers above the silicon substrate along its rim and in eight 10 micrometer wide tunnels which radiate outward from the well. The areas between the tunnels are in contact with the substrate and support the canopy. The tunnels very effectively prevent escape by the neurons.

Packaging of the chip involves lead bonding to connect the electrode pads to a standard 24-pin chip carrier and the attachment of a culture dish to the carrier. The final assembly is shown in Fig. 18a, where the scale bar is $1 \mathrm{~cm}$ long. Figure 18b shows a successfully grown cultured network, eight days in culture, with twelve neurons growing our of wells [12]. 

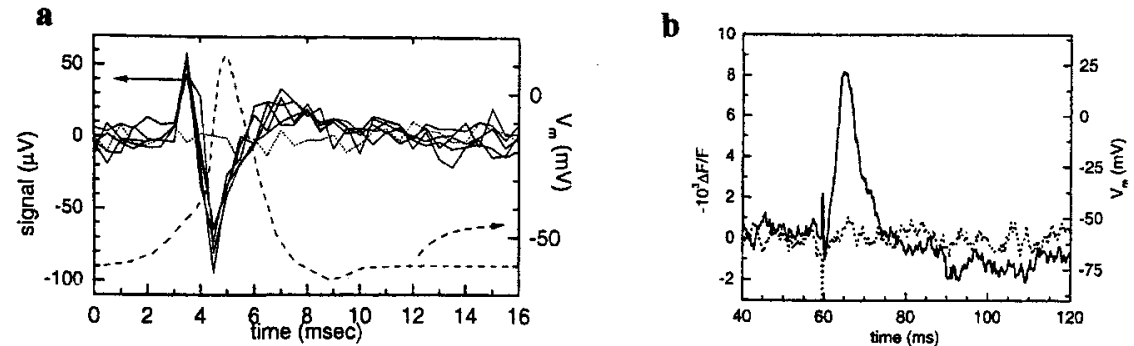

FIGURE 19. Electrical recordings of action potentials from a neurochip and dye recording from a stimulated cell.

Figure 19a shows recodings made from one cell in a well which was part of a spontaneously active network. The dashed curve shows the shape of an intracellular recording and the other traces show five action potentials aligned with their peaks at the same time. One trace shows the background noise level, and it can be seen that the signal/noise ratio is very high. There is a positive-going initial phase which we believe is from the synaptic excitation of the neuron, and a larger negative phase which coincides with the rapid rise of the action potential and the large inward sodium channel current. In order to illustrate that the well electrode can be used for stimulation as well as recording, we stimulated a neuron while recording from it

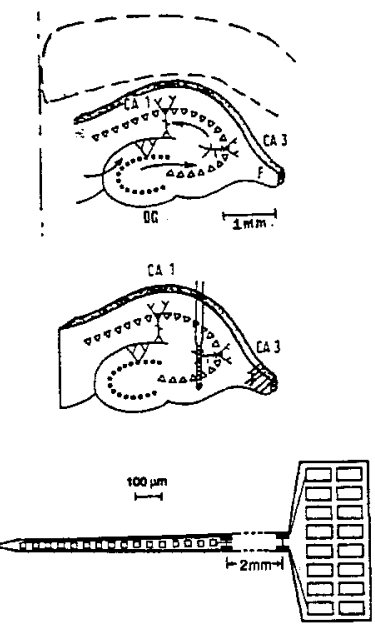

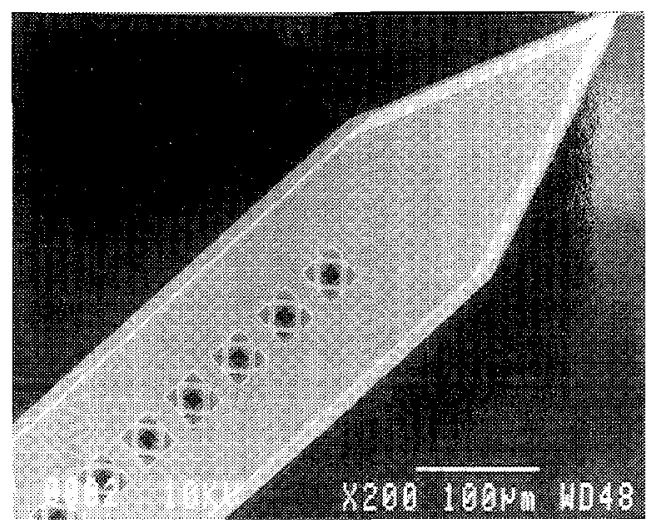

FIGURE 20. The concept of the cultured neuron probe, and a test in hippocampus. Scanning micrograph of a probe tip region. 


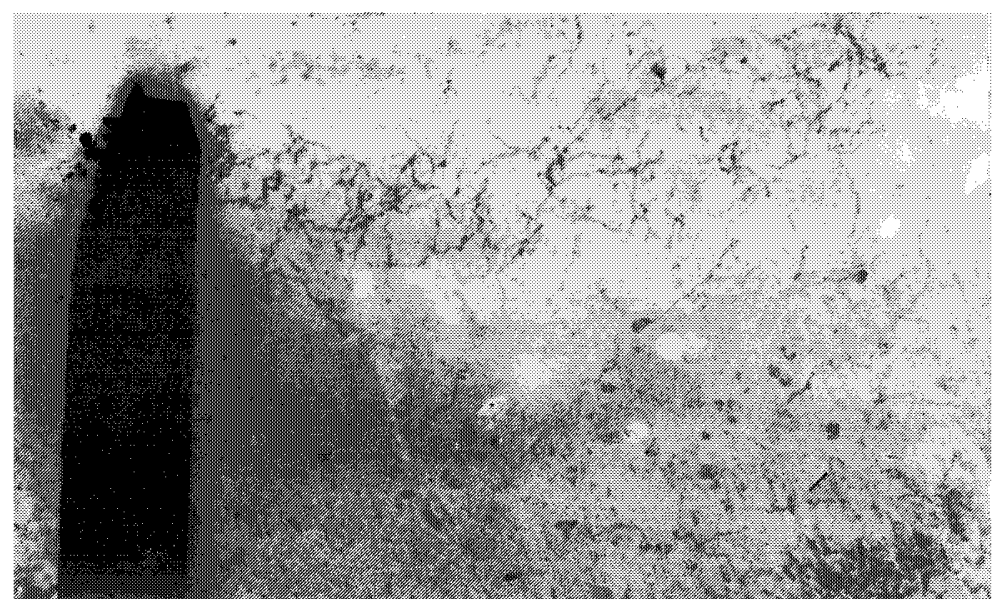

FIGURE 21. Outgrowth of stained cholinergic axons from septal neurons in a probe.

with a voltage sensitive dye. Figure $19 \mathrm{~b}$ shows such a recording, as well as a noise trace when the stimulus was below threshold.

It is clear that the neurochip functions well for both stimulation and recording of action potentials. In addition, voltage-sensitive dyes can be used to record subthreshold synaptic potentials at the cell body. However, our goal of being able to study development and plasticity of small networks is still not quite reached. Brain neuron cultures need glial cells in contact with the neurons for long term survival. The culture shown in Figure 18b, grown with medium that does not support glia, will not survive for more than a few days more, and for our studies we want cultures that grow and develop for two to three weeks. However, glial cells in such a culture can grow over the wells, and if that occurs we find that neurons can escape out the central hole. Our efforts are now focussed on developing a system where glial growth is controlled so as to ameliorate that problem.

The neurochip was developed along with what we have called a "cultured neuron probe", and because of the possible uses of the probe the work was supported by the NIH neural prosthesis program. The concept of the probe is illustrated in figure 20a, which diagrams a silicon structure with sixteen wells along a thin shaft. The probe is designed to be inserted into a brain, with its neurons connected to external circuitry by leads attached to pads on the probe handle. For initial experiments, we chose insertion of a probe loaded with hippocampal cells into a rat hippocampus. That is diagrammed in Figure 20a. The idea of the probe is that the neurons in the wells will grow out and integrate into the host nervous system, thereby creating a two-way communication link. The potential advantage of the probe is that the connection is far more cell-specific and also less invasive than a metal electrode which would otherwise be used. From the standpoint of a 
prosthesis, the probe might be used for driving sensory cortex with external signals or for recoding cortical signals for use in controlling an external system. Examples might be a visual prosthesis with probes in visual cortex, or a limb controller driven by signals recorded in motor cortex.

Figure 20b shows a silicon probe 16 micrometers thick and about 100 micrometers wide. Initial experiments have been done in collaboration with the Buzsaki lab at Rutgers to explore the possibility of probe neurons integrating into a host system. Cholinergic septal neurons which normally innervate the hippocampus were loaded into probes which were inserted into rat hippocampi. After two months, brains was fixed and sectioned, and a stain which selectively darkens cholinergic processes was used to look for outgrowth. Figure 21 shows such growth. The probe tip region is at the left in the figure, and stained processes several hundred micrometers long can be seen. This kind of result was however only seen very rarely, and work remains to be done to understand how to better promote survival, growth, and integration of probe neurons.

This concludes a review in which I have tried to present some examples that indicate the potential for using microstructures in neurobiology. My hope is that it can stimulate others to create new ideas and join in this very interesting work.

\section{REFERENCES}

1. Pine, J., J. Neurosci. Meth. 2, 19-31 (1980).

2. Thomas, C. A., Jr. et al, Exp. Cell Res. 74, 61-66 (1972).

3. Gross, G. W. et al, IEEE Trans. BME 26, 273-279 (1979).

4. Maeda, E. et al, Euro. J. Neurosci. 10, 488-498 (1998).

5. Jimbo, Y. et al, Biophys. J. 76, 670-678 (1999).

6. Kamioka, H. et al, Cellular Eng'g 2, 148-153 (1997).

7. Regehr, W. G. et al, J. Neurosci. Meth. 30, 91-106 (1989).

8. Fromherz, P. et al, Science 252, 1290-1293 (1991).

9. Meister, M., Pine, J. and Baylor, D.A., J. Neurosci. Meth. 51, 95-106 (1994).

10. Welsh et al, Neuron 14, 697-706 (1995).

11. Regehr, W. G., Pine, J. and Rutledge, D. B., IEEE Trans. BME 35, 023-1031 (1988).

12. Maher, M. P., et al, J. Neurosci. Meth. 87, 45-56 (1999). 